\title{
VISIÓN DE LA EDUCACIÓN Y EL ESTILO DE ENSEÑANZA DE LOS DOCENTES DE LA FACULTAD DE EDUCACIÓN DE LA UNIVERSIDAD NACIONAL DEL CENTRO DEL PERÚ
}

\section{VISION OF EDUCATION AND TEACHERS TEACHING STYLE OF THE EDUCATION FACULTY AT THE UNIVERSIDAD NACIONAL DEL CENTRO DEL PERU}

\author{
Jesús Tello Yance, Pedro Barrientos Gutiérrez \\ Tello Matril, Ladiska, Tello Matril, Gabriela
}

\section{RESUMEN}

El presente trabajo trata sobre la visión de la educación y los estilos de enseñanza de los docentes en opinión de los estudiantes, el propósito de la investigación fue establecer si ¿Existe correlación entre la visión de la educación y el estilo de enseñanza de los docentes de la Facultad de Educación de la Universidad Nacional del Centro del Perú?, el nivel es el descriptivo y el tipo aplicado, el método fue el hipotético deductivo con un diseño transeccional. La población estuvo constituida por 80 docentes de la Facultad de Educación de la UNCP y la muestra por 60 , seleccionados por la fórmula de regla de tres simple. Para la recolección de datos se empleó la encuesta. Los resultado mostraron que en la mentalidad de los docentes predomina la visión mecanicista de la educación, educación vista como fragmentada, mecánica, reduccionista; El desempeño en los estilos de enseñanza de los docentes es regular, subsiste estrategias conductista y tradicional en su enseñanza y Existe una correlación baja entre la visión de la educación y los estilos de enseñanza de los docentes en la opinión de los alumnos, por lo tanto subsistiría en la mentalidad de los docentes el paradigma conductista y mecanicista de la educación.

Palabras Clave: Educación y enseñanza

\begin{abstract}
The present paper deals with the vision of education and teaching styles of teachers in the view of students, the purpose of the research was to determine if there is a correlation between the vision of education and the teaching style of the teachers of the Faculty of education of the Universidad Nacional del Centro del Peru?, the level is the descriptive and the applied type, the method was the hypothetical deductive transectional design. The population was made up of 80 teachers of the Faculty of education of the UNCP and the sample by 60 , selected by the formula of simple rule of three. The survey was used for data collection. The results showed that the mechanistic vision of education, predominates in the mentality of teachers view education as fragmented, mechanical, reductionist; Performance in the teaching is teaching regular styles, there is traditional and behavioral strategies in their teaching and there is a low correlation between the vision of education and teaching styles of teachers in the opinion of the students, therefore remained in the minds of teachers education behavioral and mechanistic paradigm.
\end{abstract}

Keywords: Education and education

\section{INTRODUCCIÓN}

El desempeño de la docencia tiene su sello característico propio frente a sus estudiantes, lo más permanente y la esencia de su vocación que refleja en su perfil humano y profesional; es decir su estilo de enseñanza que tiene que ver con el rol que cumple cada maestro durante su sesión de aprendizaje para lograr los mejores resultados en sus educandos. Este rol está referido a qué debo hacer yo y mis educandos en el aula para asegurar que los pasos pedagógicos previstos cumplan ofreciendo un ambiente agradable y divertido para el aprendizaje. Ello implica tener una visión de la educación que permita tener un enfoque, 
concepción, teoría y paradigma educativo para actuar en la formación integral de los estudiantes.

Los cambios que se está dando en estos últimos años es un proceso de cambio de paradigma que transita desde la ciencia mecanicista de los siglos XVII a XX hasta la nueva visión iniciada con la física quántica y que continúa con los desarrollos tales como el modelo holográfico del cerebro de Kart $\mathrm{H}$. Pribram, (1991) la teoría del caos de llyaPrigogine (1984) , la resonancia mórfica de Rupert Sheldrake (1990) , la hipótesis Gaia de James Lovelock (1985) , el orden implicado de David Bohn (1959, la economía ecológica de Hzel Henderson (1998) , la medicina holista de Larry Dossey (1989) , etc. El conjunto de estos desarrollos conforman lo que se conoce como los nuevos paradigmas, representan un cambio de paradigma, una revolución en el conocimiento y una alternativa poderosa para que la humanidad pueda enfrentar eficazmente los retos del siglo XXI.

El presente trabajo trata sobre la visión de la educación y los estilos de enseñanza de los docentes en opinión de los estudiantes, en el mundo de la educación actual subsiste una mixtura de ideas, opiniones, enfoques y paradigmas, en educación no se ha producido la revolución del pensamiento. En el mayor porcentaje de los docentes subsisten esquemas y paradigmas tradicionales de entender la educación.

Según los teóricos y estudiosos de la materia, se puede aseverar que la visión del mundo que predomina actualmente en nuestra cultura nació en el siglo XVII con Francis Bacon. Los postulados teóricos han cambiado de nombre -empirismo, racionalismo, positivismo, etc.- sin embargo, la esencia es la misma: tener una visión mecanicista del mundo. Ante la subsistencia de la visión, según los intelectuales modernos, surge la imperiosa necesidad de buscar alternativas que ayuden a producir conocimientos, estos indudablemente deben tener la característica de científicos, comprobado está que la visión racionalista y su método no es el camino más adecuado para la producción científica, eso se debe a que deja fuera el estudio de la conciencia en el proceso mismo de producción de ciencia. "La ciencia es posible por la existencia de la conciencia, si no existiera la conciencia no se podría producir ciencia. Desde la visión holista, la nueva ciencia construye nuevos conceptos para estudiar las experiencias de los organismos con conciencia. Esto incluye, de manera central, la experiencia espiritual humana. La nueva ciencia con conciencia percibe el universo no como un conjunto de objetos, sino como una comunidad de sujetos» Larry Dossey (1989 ). Indiscutiblemente, debemos abordar en el ámbito educativo el asunto de la visión holista, ya que debe ser vista como una alternativa para superar los paradigmas dogmáticos y cientificistas. Aquí se contrasta estas ideas de la educación con los resultados de la opinión de los estudiantes de la Facultad de Educación.

\section{MÉTODO}

La investigación estuvo enmarcada dentro de la investigación descriptiva; cuyo propósito fue determinar la relación que existe entre dos o más variables en un contexto particular y pretende ver si están o no relacionadas en los mismos sujetos y, después analizar la relación (Hernández, Fernández y Baptista; 1999)

\section{Diseño metodológico de la investigación}

El diseño metodológico de la investigación, responde al diseño descriptivo correlacional, porque no existió manipulación activa de alguna variable, ya que se trató de identificar y establecer la relación que existe entre las dos variables: Visión de la educación y los estilos de enseñanza de los docentes de la Facultad de Educación de la UNCP (Barrientos; 2006 p 61). Cuyo esquema es el siguiente:

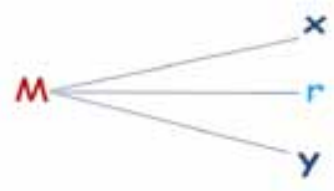

Dónde:

$M=$ Muestra

$x=$ Visión de la educación

$r=$ Relación (Correlación de Pearson)

$y=$ Estilos de enseñanza 
El presente trabajo de investigación se ha realizado mediante el método No experimental y de manera específica por el método hipotético deductivo. Según Sierra (2002:30), se entiende por método, como procedimiento, está constituido por las etapas generales de actuación que forma su contenido y por las técnicas y procedimientos concretos, operativos, para realizar en un caso determinado las fases generales de actuación en cuestión.

El método hipotético deductivo, según Sierra (2002:20), en cuanto se basa en la formulación de problemas, cuestiones o interrogantes sobre la realidad y en adelantar conjeturas soluciones probables a dichas cuestiones. Por la naturaleza de la investigación, por el tema y el problema a tratar, el método hipotético es el más adecuado y pertinente.

\section{Población y muestra}

La población de estudio estuvo constituida por todos los docentes y estudiantes de la Facultad de Educación de la UNCP. La muestra de estudio ha sido seleccionada mediante la técnica de muestreo no probabilística, a criterio de los investigadores, siendo 60docentes seleccionados y 107 estudiantes de X semestre de cada Escuela Académico Profesional de la Facultad de Educación de la UNCP.

Muestra seleccionada de estudiantes de las carreras profesionales de la Facultad de Educación

\begin{tabular}{|c|c|c|}
\hline $\mathrm{N}^{\circ}$ & Carreras profesionales & Estudiantes \\
\hline 1 & Educación primaria & 28 \\
\hline 2 & Educación inicial & 16 \\
\hline 3 & $\begin{array}{l}\text { Filosofía, ciencias } \\
\text { sociales }\end{array}$ & 10 \\
\hline 4 & Matemática física & 15 \\
\hline 5 & $\begin{array}{l}\text { Lenguas y } \\
\text { comunicación }\end{array}$ & 9 \\
\hline 6 & $\begin{array}{l}\text { Ciencias naturales y } \\
\text { ambiente }\end{array}$ & 11 \\
\hline \multirow[t]{2}{*}{7} & $\begin{array}{l}\text { Educación física y } \\
\text { psicomotricidad }\end{array}$ & 18 \\
\hline & Total & 107 \\
\hline
\end{tabular}

Técnicas e instrumentos de recolección de datos

En la presente investigación, los datos se obtuvieron en dos fases: la primera consiste en el trabajo de campo se efectúa el encuentro entre el investigador y el fenómeno estudiado, la segunda fase es el trabajo en gabinete consiste en el análisis de los datos mediante el uso de la estadística.

La recopilación de datos se ha realizado mediante la técnica de la encuesta, para ello se elaboró como instrumentos los cuestionarios a partir de los indicadores de las variables de investigación. La confiabilidad del instrumento fue determinada con el Alfa de cronbach, el resultado fue mayor que 0,60 .

Los datos obtenidos a través de la aplicación delos instrumentos se procesaron por medio de las técnicas estadísticas descriptivas, de correlación e inferencial.

\section{Presentación de los resultados}

Interpretación de los resultados escalas de ponderación de la visión de la educación

Tabla 1. Estilos de enseñanza predominante en los docentes de la faculta de educación

\begin{tabular}{|c|c|c|c|c|c|c|c|}
\hline & $\begin{array}{c}\text { Estilos de } \\
\text { Enseñanza } \\
: \\
\text { Educación } \\
\text { Inicial } \\
\end{array}$ & $\begin{array}{c}\text { Estilos de } \\
\text { Enseñanza } \\
: \\
\text { Educación } \\
\text { Primaria }\end{array}$ & $\begin{array}{c}\text { Estilos } \\
\text { de } \\
\text { Enseñ } \\
\text { anza: } \\
\text { Cienci } \\
\text { as } \\
\text { Social } \\
\text { es } \\
\end{array}$ & $\begin{array}{l} \\
\text { Estilos de } \\
\text { Enseñanza: } \\
\text { Lenguas y } \\
\text { Literatura }\end{array}$ & $\begin{array}{l} \\
\text { Estilos de } \\
\text { Enseñanza: } \\
\text { Ciencias } \\
\text { Naturales } \\
\end{array}$ & $\begin{array}{l}\text { Estilos } \\
\text { de } \\
\text { Enseña } \\
\text { nza: } \\
\text { Matemá } \\
\text { tica e } \\
\text { Informát } \\
\text { ica } \\
\end{array}$ & $\begin{array}{c}\text { Estilo } \\
\text { s de } \\
\text { Ense } \\
\text { ñanz } \\
\text { a: } \\
\text { Educ } \\
\text { ación } \\
\text { Fisic } \\
\text { a } \\
\end{array}$ \\
\hline Válidos & 4 & 4 & 4 & 4 & 4 & 4 & 4 \\
\hline $\begin{array}{l}\text { Perdido } \\
\mathrm{s}\end{array}$ & 0 & 0 & 0 & 0 & 0 & 0 & 0 \\
\hline Media & 3.50 & 3.00 & 3.00 & 3.00 & 3.00 & 3.50 & 3.00 \\
\hline
\end{tabular}

Leyenda: Escala de cualificación de los estilos de enseñanza

$1 \mathrm{mal}$

2 insuficiente

3 regular

4 bien

5 con suficiencia 
Esta escala permite determinar el nivel de los estilos enseñanza que predomina en los docentes de la Facultad de Educación.

Cuadro 1. Consolidado de medias de los resultados de los estilos de enseñanza de los docentes de la Facultad de Educación de la UNCP.

\begin{tabular}{|c|c|c|c|}
\hline \multirow{2}{*}{$\begin{array}{c}\text { Escuelas académicos } \\
\text { profesionales }\end{array}$} & & \multicolumn{2}{|c|}{ Estilo de enseñanza } \\
\hline & & $X$ & Cualificación \\
\hline EAP. Educación inicial & & 3,5 & Regular \\
\hline EAP. Educación primaria & & 3 & Regular \\
\hline $\begin{array}{l}\text { EAP. Ciencias sociales } \\
\text { relaciones humanas }\end{array}$ & y & 3 & Regular \\
\hline $\begin{array}{l}\text { EAP. Lengua literatura } \\
\text { comunicación }\end{array}$ & $\mathrm{y}$ & 3 & Regular \\
\hline EAP. Ciencias naturales & & 3 & Regular \\
\hline $\begin{array}{l}\text { EAP. Matemática } \\
\text { informática }\end{array}$ & e & 3,5 & Regular \\
\hline EAP. Educación física & & 3 & Regular \\
\hline ESTILO & & 3 & Regular \\
\hline
\end{tabular}

Este cuadro describe los estilos predominantes de los docentes de las diferentes escuelas profesionales de la Facultad de Educación.

El estilo de enseñanza que predomina en los docentes es de nivel regular, significa que los docentes que enseñan las materias de su especialidad en todas las carreras de educación su desempeño no son tan óptimos en las aulas de clase, existe repetición frecuente de los estilos de enseñanza con mayor tiempo de permanencia.

Cuadro 2 . Correlación entre la visión de la educación y el estilo de enseñanza de los docentes.

\begin{tabular}{|c|c|c|c|}
\hline & & $\begin{array}{l}\text { Visión de la } \\
\text { Educación } \\
\text { Facultad de } \\
\text { Educación }\end{array}$ & $\begin{array}{c}\text { Estilos de } \\
\text { Enseñanza de los } \\
\text { Docentes Facutad de } \\
\text { Educación }\end{array}$ \\
\hline \multirow{6}{*}{$\begin{array}{l}\text { Visión de la } \\
\text { Educación } \\
\text { Facultad de } \\
\text { Educación } \\
\text { Estilos de } \\
\text { Enseñanza de } \\
\text { los Docentes } \\
\text { Facultad de } \\
\text { Educación }\end{array}$} & $\begin{array}{l}\text { Correlación de } \\
\text { Pearson }\end{array}$ & 1 & .441 \\
\hline & Sig. (bilateral) & & .322 \\
\hline & $\mathrm{N}$ & 7 & 7 \\
\hline & $\begin{array}{l}\text { Correlación de } \\
\text { Pearson }\end{array}$ & .441 & 1 \\
\hline & Sig. (bilateral) & .322 & \\
\hline & $\mathrm{N}$ & 7 & 7 \\
\hline
\end{tabular}

Correlaciones entre la visión de la educación y estilos de enseñanza de los docentes

Tabla:

Este cuadro muestra una correlación baja entre la visión de los educación y los estilos de enseñanza en los docentes, la correlación es 0,44 , esta cifra es menor a la ponderación máxima de correlación.

\section{Relación estadística de muestras relacionadas}

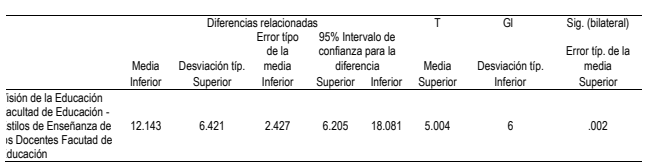

La tabla 1, presenta los resultados de la correlación entre la visión de los educación y estilos de enseñanza de los docentes.

\section{Discusión de los resultados}

De acuerdo al cuadro 1, se observa que el estilo de enseñanza de los docentes de la Facultas de Educación de la UNCP, en los diferentes componentes cualifican su desempeño como regular; es decir, el rol que cumplen los docentes en el aula durante el proceso de enseñanza y aprendizaje 0 cómo conducen 0 dirigen el aprendizaje no garantiza los mejores resultados en el aprendizaje de los estudiantes en las diferentes Escuelas Académicos Profesionales.

Existe una correlación baja entre la visión de la educación y los estilos de enseñanza de los docentes, esto significaría que en los docentes subsiste el paradigma mecanicista y fragmentario de la visión de la educación, además mantendría un estilo tradicional y conductista en la enseñanza. Frente a estos paradigmas se debe optar por un paradigma holística que según, El sociólogo alemán JurgenHabermas habla acerca de cómo la racionalidad instrumental impide al individuo tener una visión holística de la realidad, en consecuencia se encuentra condicionado e impedido para contar con dicha visión. En este sentido "considera insuficiente interpretar a la modernidad como un proceso de racionalización, cuando ésta última se remite exclusivamente a la razón funcional y deja de lado todas las formas de acción social-acción comunicativa, Habermas (2003).

La práctica pedagógica, vista desde esta perspectiva holista, no permite la comparación entre los sujetos ya que dicha comparación entorpece el aprendizaje, fomenta el desinterés por el estudio y 
destruye la autoestima del individuo. Si evitamos este tipo de acciones, entonces crearemos en nuestros alumnos una cultura de ganadoresganadores. En la educación holista, aprender es un concepto que adquiere una connotación especial, difiere mucho del concepto que se tiene en la educación mecanicista, desde la educación holista, «aprender es un proceso que implica muchos niveles de la conciencia humana como el afectivo, físico, social y espiritual, rebasando por completo lo puramente cognitivo y memorístico. Aprender se convierte en proceso creativo y artístico; aprender a aprender es el propósito de la educación para el siglo XXI, Dossey (1989).

\section{CONCLUSIONES}

- En la mentalidad de los docentes predomina la visión mecanicista de la educación, educación vista como fragmentada, mecánica, reduccionista.

- El desempeño en los estilos de enseñanza de los docentes es regular, subsiste estrategias conductista y tradicional en su enseñanza.

- Existe una correlación baja entre la visión de la educación y los estilos de enseñanza de los docentes en la opinión de los alumnos, por lo tanto subsiste en la mentalidad de los docentes el paradigma conductista y mecanicista de la educación.

\section{REFERENCIAS BIBLIOGRÁFICAS}

1. Aramburu Oyarbide, Mikel. Relaciones entre el desarrollo operativo, las preconcepciones y el estilo Cognitivo.

2. Barrientos Gutiérrez, Pedro. Visión Holista de la Educación. Hacia un aprendizaje con rostro humano. Editorial Ugraph S.A.C. Lima - Perú, 2007.

3. Cordamone Ricardo. Neuropsicología del pensamiento: un enfoque histórico- cultural. Buenos Aires-Argentina, 2004

4. Duval, Raymundo. Semiosis y pensamiento humano, Ediciones Scientifiqueseuropieennses, Colombia, 1999.

5. Ferreiro, Ramón F. Implicaciones educativas de los estudios sobre el pensamiento, universidad la Salle México, 2007.

6. Grace J. Craig, Anita; Woolfolk. Manual de Psicología y desarrollo educativo, Editorial Prentue-hall Hispanoamérica, 1990.

7. Hernández Sampiere, Roberto, Ferández Collado, Carlos, Baptista Lucio, Pilar. Metodología de la Investigación, McGRW-HILL INTERAMERICANA DE México, S.A.,1995.

8. Kurlond, Daniel J. Lectura Crítica versus pensamiento crítico simbiótico, 2005.

9. Mizraji, Eduardo. Las investigaciones sobre las bases biológicas del pensamiento humano, Instituto de Biología - facultad de Ciencias. Universidad de la República-Uruguay, 2002.

10. Maniatan, Hugo. Evolución del pensamiento, www.mariatan.com, 1994.

11. Montoya, Víctor. Lenguaje y Pensamiento, www.geocities.com, en su artículo Lenguaje y Pensamiento, 2004.

12. Pérez Ángel, Pérez Hilda. El desarrollo del pensamiento lógica en las clases de física, Cuba, 2002.

13. Kerlinger, Fred N. Investigación del comportamiento, McGRW-HILL INTERAMERICANA DE México, S.A., 1994.

15. Petrovski, A. Psicología Evolutiva y Pedagógica, Editorial Progreso Moscú, 1979.

16. Rivero, Norma Rosa. Cognición y estilos de pensamiento. Caracas USR-Línea -I., 2000.

17. Sáenz Vigo, Wilfredo. Investigación- Guía I, Lima-Perú, 2006.

18. Salkind, Neul J. Métodos de investigación, Prentice Hall, México, 1997 - 1999.

19. Sierra Bravo, Restituto. Tesis doctorales y trabajos de investigación científica, Paraninfo Thonson Editores, España, 2002. 
20. Sierra Bravo, Restituto. Técnicas de investigación social, Paraninfo Thonson Editores, España, 2001.

21. Smirnov, A. Leontiev, A. Psicología Grijalbo, México, 1960.

22. Van-Halen Juan. Implicaciones de la ponencia sobre la situación de la enseñanza científica en la educación secundaria, 2004.
23. Vásquez Gómez, Gonzalo. Pedagogía Cognitiva. Ediciones: Universidad de Salamanca, España.

24. Vásquez Rosado, Angie. Vygotsky y Luria. Dos aliados, dos amigos, dos vidas: un acuerdo teórico- práctico sobre la mente y el protagonismo de lo social. UIPR, Puerto Rico, 2006. 